\title{
Heisenberg Spin Fluid in an External Magnetic Field
}

\author{
F. Lado* and E. Lomba \\ Instituto de Química Física Rocasolano, Consejo Superior de Investigaciones Científicas, Serrano 119, E-28006 Madrid, Spain
}

(Received 15 October 1997)

\begin{abstract}
We develop a general method to study inhomogeneous liquids in an external field using orthogonal polynomials tailored to the one-body density. The procedure makes integral equation calculations of these systems no more difficult than those of ordinary homogeneous molecular fluids. We apply this method to the ferromagnetic Heisenberg spin fluid in a magnetic field using a "reference" version of the Zerah-Hansen closure, with no further approximations. Comparison with simulation shows this integral equation procedure yielding nearly exact results. [S0031-9007(98)05886-4]
\end{abstract}

PACS numbers: 64.60.-i, 61.20.Gy, 75.10.-b, 75.30.-m

The Gibbsian $N$-body density function of a Hamiltonian $H_{N}$ that is rotationally and translationally invariant must itself be rotationally and translationally invariant, as must then also be all reduced $n$-body density functions of this Hamiltonian. In particular, the one-body density is a constant. An external field destroys this homogeneity, producing anisotropy or nonuniformity in the one-body density $[1,2]$, and so makes necessary the joint calculation of the coupled one-body and two-body density functions. A striking if familiar example of the response of a bulk system to an external field is ferromagnetism. In this paper, we shall use this particular case to present a general procedure to compute the coupled one-body and two-body density functions of an inhomogeneous classical fluid in an external field. Remarkably, the procedure is no more difficult to carry through than similar calculations for ordinary homogeneous systems.

Perhaps the simplest model of a disordered continuum system exhibiting ferromagnetic behavior is a fluid of hard spheres with embedded Heisenberg spins described using classical statistical mechanics [3-6]. This interaction potential is clearly inadequate to model ferrofluids, in particular at low concetrations, where the dipole-dipole interaction is dominant, but it is however relevant for a description of ferromagnetism in undercooled liquid metal alloys [7]. The Heisenberg spin fluid in an external magnetic field $\mathbf{B}_{0}$ is succinctly defined by the canonical excess partition function

$$
\begin{aligned}
Z^{\mathrm{ex}}= & \frac{1}{(4 \pi V)^{N}} \int \prod_{j=1}^{N}\left[d \mathbf{r}_{j} d \omega_{j} f_{0}\left(\omega_{j}\right)\right] \\
& \times \exp \left(-\beta \sum_{i<j}\left[u_{0}\left(r_{i j}\right)+u_{s s}\left(r_{i j}, \omega_{i}, \omega_{j}\right)\right]\right) .
\end{aligned}
$$

Here $u_{0}(r)$ is the hard sphere potential for spheres of diameter $\sigma$ while $u_{s s}\left(r, \omega_{1}, \omega_{2}\right)=-J(r) \hat{\mathbf{s}}_{1} \cdot \hat{\mathbf{s}_{2}}$ for $r>$ $\sigma$ is the Heisenberg spin-spin interaction, with

$$
\beta J(r)=K \frac{e^{-\kappa(r / \sigma-1)}}{r / \sigma},
$$

where $\beta=1 / k_{B} T$. The orientations $\omega=(\theta, \phi)$ of the unit spins $\hat{\mathbf{s}}$ are referred to the uniform field $\mathbf{B}_{0}$, which defines the $z$ direction. The contribution of the spin-field interactions to the total configurational energy in Eq. (1) enters through the normalized one-body orientational distribution $f_{0}(\omega)$ of a noninteracting spin system,

$$
f_{0}(\omega)=\frac{e^{\beta \mu B_{0} \cos \theta}}{\sinh \left(\beta \mu B_{0}\right) / \beta \mu B_{0}},
$$

where $\mu$ is the spin dipole moment. In Eq. (2), the dimensionless coupling strength $K$ may be read as the inverse reduced temperature, $K=1 / T^{*}$, while $\kappa$ is a dimensionless range parameter.

The key quantities needed for a complete magnetic and thermodynamic description of this system are the onebody and two-body density functions,

$$
\begin{gathered}
\rho^{(1)}(\mathbf{r}, \omega)=\frac{\rho}{4 \pi} f(\omega), \\
\rho^{(2)}\left(\mathbf{r}, \omega, \mathbf{r}^{\prime}, \omega^{\prime}\right)=\frac{\rho^{2}}{(4 \pi)^{2}} f(\omega) f\left(\omega^{\prime}\right) g\left(\left|\mathbf{r}-\mathbf{r}^{\prime}\right|, \omega, \omega^{\prime}\right),
\end{gathered}
$$

where $\rho=N / V$ is the density, $f(\omega)$ the one-body orientational distribution in the interacting fluid, and $g\left(r, \omega, \omega^{\prime}\right)$ the pair distribution function of the inhomogeneous spin system in an external magnetic field.

The basic equations that determine the distribution functions $f(\omega)$ and $g\left(r, \omega, \omega^{\prime}\right)$ are well known [8,9]. The one-body density can be differentiated with respect to $x=\cos \theta$ to give

$$
\begin{aligned}
\frac{d}{d x} \ln \left[\frac{f(\omega)}{f_{0}(\omega)}\right]= & -\frac{\rho}{4 \pi} \int d \mathbf{r} d \omega^{\prime} f\left(\omega^{\prime}\right) \\
& \times g\left(r, \omega, \omega^{\prime}\right) \frac{d \beta u_{s s}\left(r, \omega, \omega^{\prime}\right)}{d x},
\end{aligned}
$$

the first member of a Kirkwood-Born-Green-Yvon hierarchy. Calculation of $f(\omega)$ from this equation requires knowing $g$. In classical liquid state theory, the pair distribution function is obtained from the Ornstein-Zernike (OZ) equation,

$$
\begin{aligned}
\gamma\left(r_{12}, \omega_{1}, \omega_{2}\right)= & \frac{\rho}{4 \pi} \int d \mathbf{r}_{3} d \omega_{3} f\left(\omega_{3}\right) \\
& \times\left[\gamma\left(r_{13}, \omega_{1}, \omega_{3}\right)+c\left(r_{13}, \omega_{1}, \omega_{3}\right)\right] \\
& \times c\left(r_{32}, \omega_{3}, \omega_{2}\right),
\end{aligned}
$$


and a closure relation. The $\mathrm{OZ}$ equation connects the indirect correlation function $\gamma=g-1-c$ with the direct correlation function $c$. The closure relation expresses $c$ back in terms of $\gamma$ and the model's pair interactions, along with an additional quantity, the so-called bridge function $b\left(r, \omega_{1}, \omega_{2}\right)$, which must be approximated [8,9].

While well known, these equations, to our knowledge, have never been solved for a fluid in an external magnetic or electric field, seemingly a formidable task. We do so here and find that, with the right tools, they are in fact no more difficult to solve than those of homogeneous molecular liquids. The "right tools" turn out to be orthogonal polynomials tailored to the specific one-body distribution of the fluid [10]. The usual approach for orientation-dependent functions such as $\gamma\left(r, \omega_{1}, \omega_{2}\right)$ is to expand in spherical harmonics $Y_{l m}(\omega)$ [11],

$$
\begin{aligned}
\gamma\left(r, \omega_{1}, \omega_{2}\right) & =4 \pi \sum_{l_{1}, l_{2}, m} \gamma_{l_{1} l_{2} m}(r) Y_{l_{1} m}\left(\omega_{1}\right) Y_{l_{2} \bar{m}}\left(\omega_{2}\right), \\
Y_{l m}(\omega) & =\frac{1}{\sqrt{4 \pi}}(-1)^{m} e^{i m \phi} P_{l m}(\cos \theta),
\end{aligned}
$$

where $P_{l m}(\cos \theta)$ is the familiar associated Legendre function, but here renormalized to two, and $\bar{m}=-m$. This expansion indeed makes the solution of the inhomogeneous liquid equations very difficult. We will instead expand in modified spherical harmonics,

$$
\begin{aligned}
\gamma\left(r, \omega_{1}, \omega_{2}\right) & =4 \pi \sum_{l_{1}, l_{2}, m} \gamma_{l_{1} l_{2} m}(r) \mathcal{Y}_{l_{1} m}\left(\omega_{1}\right) \mathcal{Y}_{l_{2} \bar{m}}\left(\omega_{2}\right) \\
\mathcal{Y}_{l m}(\omega) & =\frac{1}{\sqrt{4 \pi}}(-1)^{m} e^{i m \phi} \mathcal{P}_{l m}(\cos \theta)
\end{aligned}
$$

The modified Legendre functions $\mathcal{P}_{l m}(\cos \theta)$ are explicitly constructed using the Gram-Schmidt method with the orthonormality condition

$$
\frac{1}{2} \int_{-1}^{1} d x f(x) \mathcal{P}_{l m}(x) \mathcal{P}_{l^{\prime} m}(x)=\delta_{l l^{\prime}},
$$

where $f(\cos \theta)$ is the one-body distribution of the fluid. For an isotropic fluid with $f(x)=1$, this construction produces the standard Legendre functions $P_{l m}(x)$ (normalized to two). For an anisotropic fluid with $f(x) \neq 1$, the difference between $P_{l m}(x)$ and $\mathcal{P}_{l m}(x)$ is crucial.

Thus, the OZ equation (7) deconvoluted by Fourier transformation and with the pair functions expanded as in Eq. (9) becomes

$$
\tilde{\gamma}_{l_{1} l_{2} m}(k)=(-1)^{m} \rho \sum_{l_{3}}\left[\tilde{\gamma}_{l_{1} l_{3} m}(k)+\tilde{c}_{l_{1} l_{3} m}(k)\right] \tilde{c}_{l_{3} l_{2} m}(k) .
$$

The significant feature here is that this $\mathrm{OZ}$ equation for an inhomogeneous fluid in an external field is now identical to that of an ordinary homogeneous fluid [11] and so, along with a closure equation, can be solved for $g\left(r, \omega_{1}, \omega_{2}\right)$ with the same familiar algorithms already used for homogeneous systems [12].

This solution for $g\left(r, \omega_{1}, \omega_{2}\right)$ is obtained using the polynomials $\mathcal{P}_{l m}(x)$ generated with the current $f(x)$. We now return to Eq. (6) and update the one-body distribution. In expanded form, this equation is

$$
\begin{gathered}
\frac{d}{d x} \ln \left[\frac{f(x)}{f_{0}(x)}\right]=\sum_{l_{1}, l_{2}, m} \xi_{l_{1} l_{2} m} \mathcal{P}_{l_{1} m}(x) \frac{d \mathcal{P}_{l_{2} m}(x)}{d x}, \\
\xi_{l_{1} l_{2} m}=-\rho \int d \mathbf{r} \sum_{l_{3}} g_{l_{1} l_{3} m}(r) u_{l_{3} l_{2} \bar{m}}(r),
\end{gathered}
$$

where the $u_{l_{1} l_{2} m}(r)$ are the (known) coefficients of the spin-spin interaction $u_{s s}\left(r, \omega_{1}, \omega_{2}\right)$, so that finally

$$
\ln f(x)=\ln f_{0}(x)+\sum_{l=0}^{\infty} a_{l} \mathcal{P}_{l 0}(x) .
$$

Here $a_{l}$ for $l>0$ is determined by numerical (Gaussian) integration of Eq. (11) and $a_{0}$ by normalization.

The iterations for $f(\omega)$ and $g\left(r, \omega_{1}, \omega_{2}\right)$ are continued until both functions are self-consistently determined. The only approximation in the calculation is the unavoidable one in every liquid state calculation to date, the representation of the bridge function $b\left(r, \omega_{1}, \omega_{2}\right)$ [8].

We can now compute the complete magnetic and thermodynamic properties of a Heisenberg spin fluid in a uniform magnetic field $\mathbf{B}_{0}=B_{0} \hat{\mathbf{k}}$. We find the longitudinal and transverse magnetic susceptibilities are given by

$$
\begin{gathered}
\chi_{z z} / \rho \beta \mu^{2}=\sigma_{x}^{2}\left[1+\rho \tilde{h}_{110}(0)+2 \frac{\langle x\rangle}{\sigma_{x}} \rho \tilde{h}_{100}(0)\right], \\
\chi_{y y} / \rho \beta \mu^{2}=\frac{1}{2}\left(1-\left\langle x^{2}\right\rangle\right)\left[1-\rho \tilde{h}_{111}(0)\right],
\end{gathered}
$$

where $\sigma_{x}^{2} \equiv\left\langle x^{2}\right\rangle-\langle x\rangle^{2}$ for $x=\cos \theta$. For isotropic systems, with $\langle x\rangle=0$ and $\left\langle x^{2}\right\rangle=1 / 3$, these susceptibility expressions reduce to familiar forms. The pressure, internal energy, and isothermal compressibility are calculated with the standard formulas of homogeneous fluids $[8,11]$, again thanks to the special orthogonality of the $\mathcal{Y}_{l m}(\omega)$.

We have obtained the distribution functions $f(\omega)$ and $g\left(r, \omega_{1}, \omega_{2}\right)$ for the Heisenberg spin fluid in a magnetic field $\mathbf{B}_{0}$ using a "reference" version of the Zerah-Hansen [13] closure (RZH); for $r>\sigma$, this is

$$
c^{\mathrm{RZH}}\left(r, \omega_{1}, \omega_{2}\right)=\left\{\exp \left(m(r)\left[-u_{s s}\left(r, \omega_{1}, \omega_{2}\right)+\gamma\left(r, \omega_{1}, \omega_{2}\right)+b_{\mathrm{HS}}(r)\right]\right)-1\right\} / m(r)-\gamma\left(r, \omega_{1}, \omega_{2}\right) .
$$

The exact relation $c\left(r, \omega_{1}, \omega_{2}\right)=-1-\gamma\left(r, \omega_{1}, \omega_{2}\right)$ holds for $r<\sigma$. This closure features a mixing function $m(r)=1-\exp (-\alpha r)$ with a parameter $\alpha$ that is fixed by requiring consistency between the virial and compressibility bulk moduli. (However, see below for the case $B_{0} \rightarrow 0$.) The reference hard sphere bridge function $b_{\mathrm{HS}}(r)$, added above by us to the $\mathrm{ZH}$ equation [13], ensures the RZH closure yields good hard sphere results in the limit of small $K$ and improves the thermodynamic results overall. To be consistent with earlier work $[3,4]$, we 
have truncated the spin-spin interaction at $r=2.5 \sigma$; this hardly affects the thermodynamics but does considerably raise the critical $K$ value.

The calculations are carried out for $\kappa=1, \rho \sigma^{3}=0.7$, $K=1 / T^{*}=0$ to 0.5 , and two values of the external field, $\beta \mu B_{0}=1$ and 0 . We find for these cases that the one-body orientational distribution function $f(x)$ continues to be well described by the functional form of $f_{0}(x)$ but with an effective field $B$,

$$
f(x)=\frac{e^{\beta \mu B x}}{\sinh (\beta \mu B) / \beta \mu B} .
$$

In the limit $B_{0} \rightarrow 0$, the effective field $B$ is zero for $K<K_{c}$ and finite for $K>K_{c}$, where $K_{c}=1 / T_{c}^{*}$ is then the computed Curie point.

For $K>K_{c}$, there is a singularity when $B_{0} \rightarrow 0$ that is different from the second order paramagneticferromagnetic transition at $K=K_{c}$. In fact, the $B_{0}=0$ line corresponds in the $B_{0}-M$ plane (where $M$ is the magnetization per particle) to the spinodal line that indicates the equilibrium between phases with positive and negative magnetization. Consequently, the transverse susceptibility $\chi_{y y}$ (but not the longitudinal component $\chi_{z z}$ ) diverges [14] as $B_{0} \rightarrow 0$, reflecting the negligible cost of rotating an ordered sample in the absence of an external field. It turns out that the optimization of $\alpha$ in the RZH closure to achieve thermodynamic consistency leads to a $\chi_{y y}$ that diverges at small but nonzero fields. This deficiency is easily cured if one relaxes the thermodynamic consistency requirement and instead optimizes the $\alpha$ parameter by displacing the divergence of $\chi_{y y}$ to the $B_{0}=0$ line. We find the thermodynamics is hardly affected by the new choice of $\alpha$.

Using Monte Carlo (MC) simulation, Nijmeijer and Weis [3] found that the paramagnetic-ferromagnetic transition for $\rho \sigma^{3}=0.7$ with the truncated potential occurs at $K_{c}=0.264 \pm 0.001$. Our calculation in the paramagnetic phase and zero-field limit yields a divergence in $\chi_{z z}$ at $K_{c}^{l}=0.2645$, in excellent agreement with the simulation value.

In Fig. 1 we show the values of the magnetization per particle $M=\langle\cos \theta\rangle$ and the second order parameter $S=\left\langle P_{2}(\cos \theta)\right\rangle$ obtained from the theory and from a standard NVT Monte Carlo simulation, using 864 particles (for $B_{0}=1$ ), 1372 and 2048 particles (for $B_{0}=0$ ), and averages over 40000 configurations. As can be seen in Fig. 1, for $B_{0}=0$ at $K=0.275$ and above both samples lead to the same results, while in the vicinity of the critical point and below there is noticeable size dependence. In the presence of an external field, $B_{0}=1$, size effects are negligible since criticality disappears and the one sample size used is sufficient. We also include in Fig. 1 some zero-field results from density functional theory in the modified mean field approximation [6] and our theoretical estimate of the critical $K_{c}^{l}$, which at the scale of the figure is indistinguishable from the finite size scaling estimate of Ref. [3]. In the vicinity of the critical temperature, one

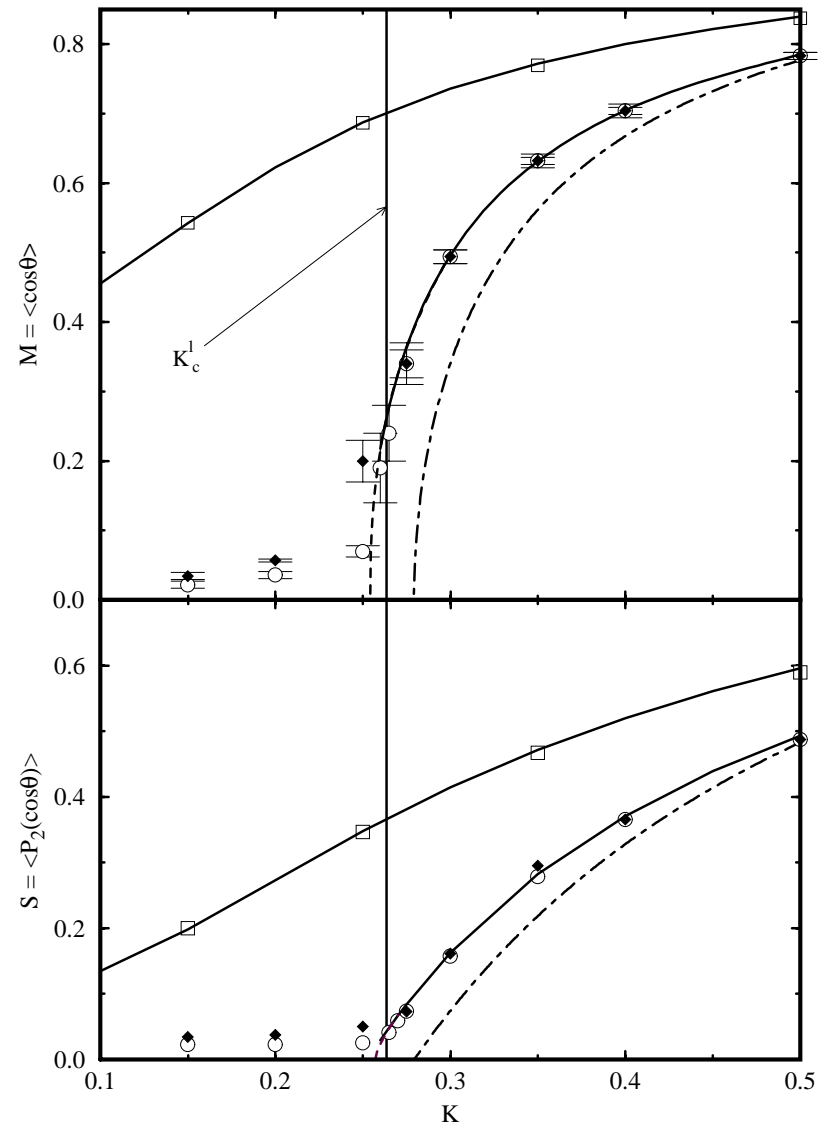

FIG. 1. Magnetization per particle $M$ and second order parameter $S$ as functions of inverse temperature $K=1 / T^{*}$ obtained from MC simulation [squares for $\beta \mu B_{0}=1$ and white circles (2048 particle sample) or black diamonds (1372 particle sample) for $\left.\beta \mu B_{0}=0\right]$ and the RZH integral equation (solid lines). The dashed lines correspond to a power law fit to the $\mathrm{RZH}$ data just above $K_{c}^{u}$. The dash-dotted curves represent density functional theory estimates.

encounters convergence difficulties in solving the integral equation as spin-spin correlations become long ranged. [Both $\chi_{z z}$ and $\chi_{y y}$ in Eqs. (14) and (15) diverge, with $\chi_{z z}$ exhibiting the characteristic $\lambda$-type divergence of secondorder transitions.] To get an estimate of the critical inverse temperature from the ordered-phase results, we fit the RZH magnetization in the vicinity of the critical temperature $(K<0.3)$ to a power law, $M=a\left(K-K_{c}^{u}\right)^{\beta}$, which leads to $a=1.694, \beta=0.397$, and $K_{c}^{u}=0.254$. The fitted value of $\beta$ is close to the value $\beta=0.387$ reported by Nijmeijer and Weis [3] and to the critical exponent of the 3D lattice Heisenberg model, $\beta=0.38 \pm 0.03$ [15]. The agreement between the critical point estimates $K_{c}^{l}=$ 0.2645 obtained in the disordered phase and $K_{c}^{u}=0.254$ obtained in the ordered phase may be regarded as good.

Computed magnetic and thermodynamic properties, including the effective field $B$, are collected in Table I. Direct comparison with Monte Carlo data is available only for the internal energy $U$ and here we find excellent agreement. That the excellent agreement between simulation and theory extends to the correlation functions can be seen 
TABLE I. Computed magnetic and thermodynamic properties of the Heisenberg spin fluid for $\rho \sigma^{3}=0.7$ and $\kappa=1$; the values of $\chi_{y y}$ at zero field for $K>K_{c}$ correspond to the last converged solution. The MC value in parentheses is a rough estimate since, due to the vicinity of the critical point, the results at this temperature are particularly sensitive to system size.

\begin{tabular}{|c|c|c|c|c|c|c|c|c|c|c|}
\hline \multirow{3}{*}{ K } & \multirow{3}{*}{$\beta \mu B$} & \multicolumn{3}{|c|}{$\beta \mu B_{0}=0$} & \multirow{3}{*}{$\chi_{y y} / \rho \beta \mu^{2}$} & \multirow{3}{*}{$\beta \mu B$} & \multicolumn{3}{|c|}{$\beta \mu B_{0}=1$} & \multirow{3}{*}{$\chi_{y y} / \rho \beta \mu^{2}$} \\
\hline & & \multicolumn{2}{|c|}{$\beta U / N$} & \multirow[t]{2}{*}{$\chi_{z z} / \rho \beta \mu^{2}$} & & & & & $\chi_{z z} / \rho \beta \mu^{2}$ & \\
\hline & & RZH & MC & & & & RZH & $\mathrm{MC}$ & & \\
\hline 0.15 & 0 & -0.034 & -0.034 & 0.877 & 0.877 & 2.018 & -0.297 & -0.298 & 0.281 & 0.540 \\
\hline 0.20 & 0 & -0.074 & -0.073 & 1.736 & 1.736 & 2.548 & -0.517 & & 0.227 & 0.618 \\
\hline 0.25 & 0 & -0.156 & $(-0.175)$ & 8.854 & 8.854 & 3.122 & -0.780 & -0.782 & 0.174 & 0.679 \\
\hline 0.30 & 1.778 & -0.597 & -0.597 & 1.655 & 8.534 & 3.712 & -1.068 & & 0.134 & 0.725 \\
\hline 0.35 & 2.637 & -1.009 & -1.008 & 0.666 & 7.733 & 4.304 & -1.367 & -1.365 & 0.106 & 0.760 \\
\hline 0.40 & 3.358 & -1.384 & -1.384 & 0.366 & 6.312 & 4.896 & -1.673 & & 0.088 & 0.786 \\
\hline 0.45 & 4.021 & -1.739 & -1.740 & 0.245 & 5.424 & 5.486 & -1.983 & & 0.075 & 0.806 \\
\hline 0.50 & 4.647 & -2.084 & -2.082 & 0.209 & 5.841 & 6.073 & -2.295 & -2.295 & 0.066 & 0.821 \\
\hline
\end{tabular}

in Fig. 2. We note that the angular projections of Eq. (9) cannot be directly compared with the standard projections typically calculated along a simulation run, since these correspond to the usual spherical harmonic basis. However, it is straightforward to recover one set of coefficients from the other if the moments of the oneparticle distribution function are known. Thus, for the $h^{110}(r)$ function plotted in Fig. 2, we have

$$
\begin{array}{r}
h^{110}(r)=3\left\{\langle x\rangle^{2} g_{000}(r)+\sigma_{x}\langle x\rangle\left[g_{100}(r)+g_{010}(r)\right]\right. \\
\left.+\sigma_{x}^{2} g_{110}(r)-\left(1-\left\langle x^{2}\right\rangle\right) g_{111}(r)\right\},
\end{array}
$$

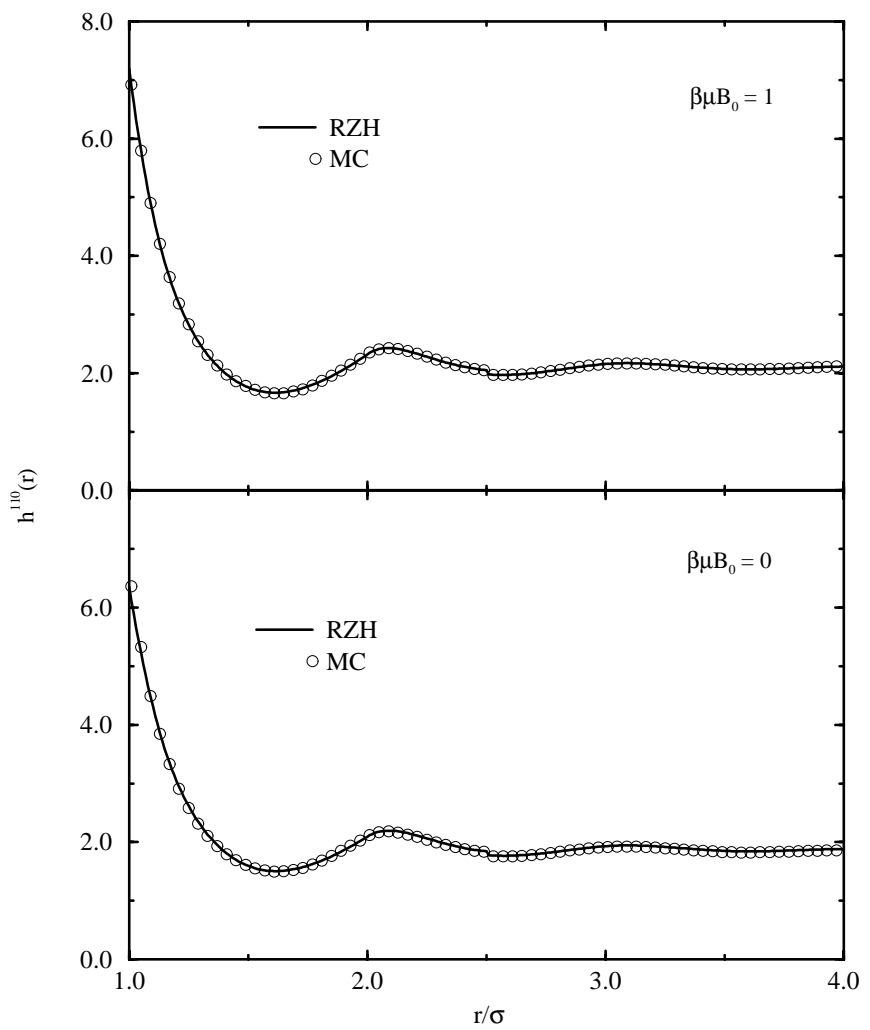

FIG. 2. The $h^{110}(r)$ angular correlation function from simulation (open circles) and the RZH integral equation (solid lines) for $K=0.5$ with and without an external magnetic field. The discontinuity at $r=2.5 \sigma$ is due to the potential truncation. which reduces to the usual $h^{110}(r)=g_{110}(r)-2 g_{111}(r)$ in the isotropic limit. This function describes most of the orientational behavior of the spin fluid, since it is the projection of $g\left(r, \omega_{1}, \omega_{2}\right)$ onto the rotational invariant $\hat{\mathbf{s}}_{1}$. $\hat{\mathbf{s}}_{2}=\cos \theta_{12}$. From Eq. (18), we have $\lim _{r \rightarrow \infty} h^{110}(r)=$ $3 M^{2}$, exactly fulfilled in our calculations by both simulated and theoretical results.

F.L. thanks the Dirección General de Investigación y Desarrollo (Spain) for sabbatical support under Grant No. SAB95-0373. This work was supported in part by the Dirección General de Investigación Científica y Técnica (Spain) under Grants No. PB94-0112 and No. PB940265 .

*On sabbatical leave from Department of Physics, North Carolina State University, Raleigh, North Carolina 27695-8202.

[1] N. D. Mermin, Phys. Rev. 137, A1441 (1965).

[2] J.R. Henderson, in Fundamentals of Inhomogeneous Fluids, edited by D. Henderson (Dekker, New York, 1992).

[3] M. J. P. Nijmeijer and J. J. Weis, Phys. Rev. Lett. 75, 2887 (1995); Phys. Rev. E 53, 591 (1996).

[4] J. J. Weis et al., Phys. Rev. E 55, 436 (1997).

[5] E. Lomba et al., Phys. Rev. E 49, 5169 (1994).

[6] J. M. Tavares et al., Phys. Rev. E 52, 1915 (1995).

[7] T. Albrecht, C. Bührer, M. Fähnle, K. Maier, D. Platzek, and J. Reske, Appl. Phys. A 65, 215 (1997).

[8] J. P. Hansen and I. R. McDonald, Theory of Simple Liquids (Academic, London, 1986).

[9] D. Henderson, in Fundamentals of Inhomogeneous Fluids, edited by D. Henderson (Dekker, New York, 1992).

[10] F. Lado, Phys. Rev. E 55, 426 (1997).

[11] C. G. Gray and K. E. Gubbins, Theory of Molecular Fluids (Clarendon, Oxford, 1984), Vol. 1.

[12] F. Lado, Mol. Phys. 47, 283 (1982).

[13] G. Zerah and J.P. Hansen, J. Chem. Phys. 84, 2336 (1986).

[14] L. D. Landau, E. M. Lifshitz, and L. P. Pitaevskii, Electrodynamics of Continuous Media (Pergamon, Oxford, 1984), 2nd ed., Sect. 46.

[15] J. J. Binney, N. J. Dowrick, A. J. Fisher, and M.E. J. Newman, The Theory of Critical Phenomena (Clarendon, Oxford, 1992), p. 82. 